\title{
Drug Resistance in Colorectal Cancer Cell Lines is Partially Associated with Aneuploidy Status in Light of Profiling Gene Expression
}

Jiao Guo, ${ }^{\dagger, \$, \beta, \#}$ Shaohang Xu, ${ }^{\S, \perp, \#}$ Xuanlin Huang,${ }^{\S, \perp}$ Lin Li,${ }^{\S, \perp}$ Congmin Zhang, ${ }^{\dagger, \$}$

Qingfei Pan, ${ }^{\dagger, \downarrow}$ Zhe Ren, ${ }^{\S, \perp}$ Ruo Zhou, ${ }^{\S, \perp}$ Yan Ren, ${ }^{\S, \perp}$ Jin Zi,,${ }^{\S, \perp}$ Lin Wu, ${ }^{\dagger,}$ Jan

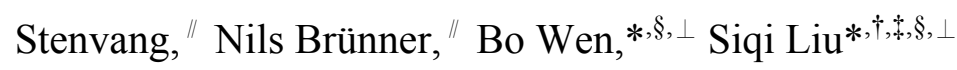

${ }^{\dagger}$ CAS Key Laboratory of Genome Sciences and Information, Beijing Institute of

Genomics, Chinese Academy of Sciences, Beijing, 100101, China

${ }^{*}$ University of Chinese Academy of Sciences, Beijing, 100049, China

${ }^{\S}$ BGI-Shenzhen, Shenzhen, Guangdong, 518083, China

" Department of Veterinary Disease Biology, Faculty of Health and Medical Sciences,

Section for Molecular Disease Biology, University of Copenhagen, Strandboulevarden 49, DK-2100 Copenhagen, Denmark

${ }^{\perp}$ China National GeneBank-Shenzhen, BGI-Shenzhen, Shenzhen 518083, China

\# These authors contributed equally to this work.

* Corresponding authors

Siqi Liu, Tel: 86-10-80485325; Fax: 86-10-80485324; E-mail: siqiliu@genomics.cn.

Bo Wen, Tel and Fax: 86-0755-25273620; E-mail: wenbo@genomics.cn. 


\section{List of supplementary components}

The article contains Supplementary Figures, Fig. S1 and S2, and Supplementary Tables, Table S1 to S5.

\begin{tabular}{|c|c|}
\hline Supplementary components & Page NO. \\
\hline Legends of Supplementary Figures. & S3 \\
\hline $\begin{array}{l}\text { Figure S1. The SNPs' MAF (minor allele frequency) ratios of the two CRC cell } \\
\text { lines with non- and drug-resistance based on WES data. }\end{array}$ & $\mathrm{S} 4$ \\
\hline $\begin{array}{l}\text { Figure S2. Cellular location with CELLO2G for the } 208 \text { genes on chromosome } 14 \\
\text { which were identified by RNAseq and categorized as phosphorylation function } \\
\text { related. }\end{array}$ & S5 \\
\hline $\begin{array}{l}\text { Table S1. The sequencing depth for the two CRC cell lines with non- and drug- } \\
\text { resistance based on NGS data by WES. }\end{array}$ & S6 \\
\hline $\begin{array}{l}\text { Table S2. Average genome copy numbers of HCT116 and LoVo derived from } \\
\text { database. }\end{array}$ & S7 \\
\hline $\begin{array}{l}\text { Table S3. Table of protein iBAQ values for HCT116 and LoVo samples. } \\
\text { (including } 2 \text { sheets) }\end{array}$ & Excel \\
\hline $\begin{array}{l}\text { Table S4. Table of iTRAQ values for HCT116 and LoVo samples. （including } 5 \\
\text { sheets) }\end{array}$ & Excel \\
\hline Table S5. The correlation of CRC cell lines and iTRAQ labelling tags. & S8 \\
\hline
\end{tabular}




\section{Legends of Supplementary Figures}

Fig S1. The SNPs' MAF (minor allele frequency) ratios of the two CRC cell lines with non- and drug-resistance based on WES data. X-axis is defined as chromosome order and Y-axis is represented by the MAF ratio of the SNPs in individual chromosomes. The MAF distributions of HCT116, HCT116-OxPt, HCT116-SN38, LoVo, LoVo-OxPt, and LoVo-SN38 cell lines were presented separately.

Fig S2. Cellular location with CELLO2G for the 208 genes on chromosome 14 which were identified by RNAseq and categorized as phosphorylation function related. 


\section{Figure S1}

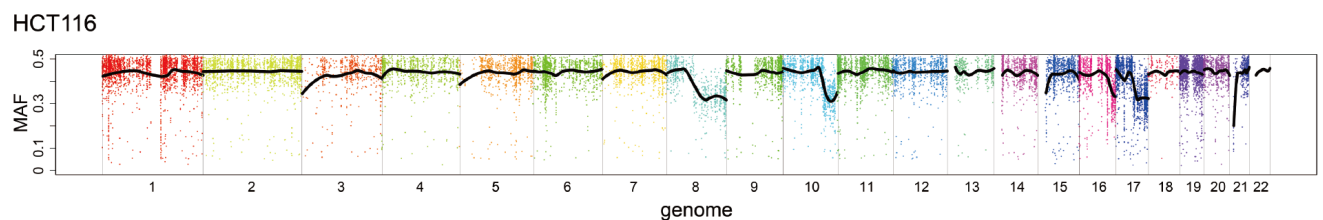

HCT116-OxPt

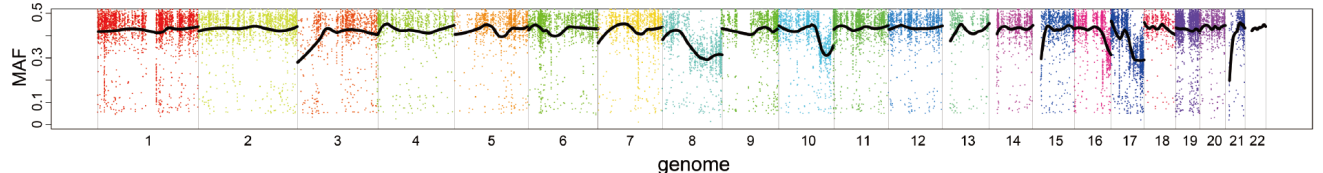

HCT116-SN38
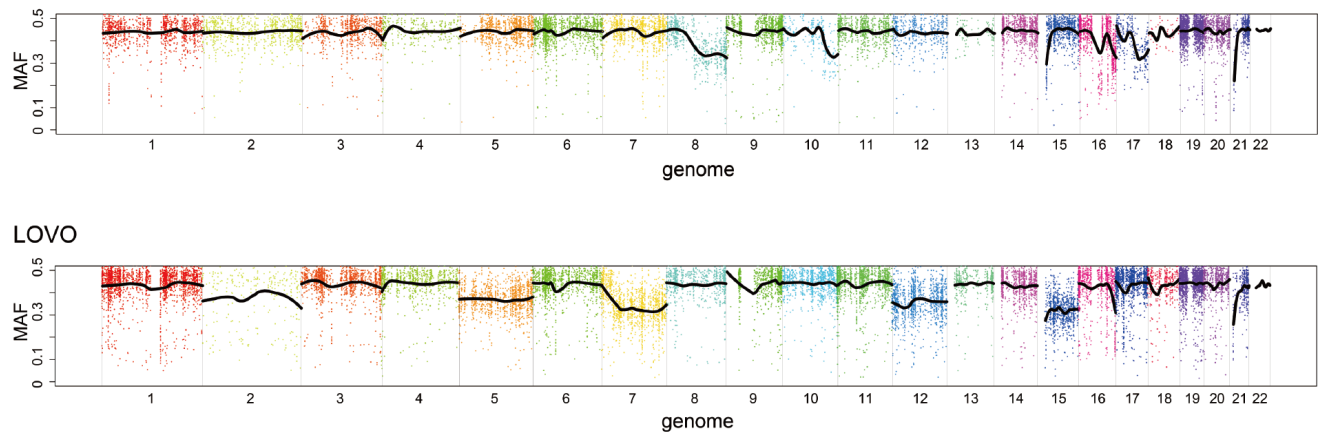

LOVO-OxPt

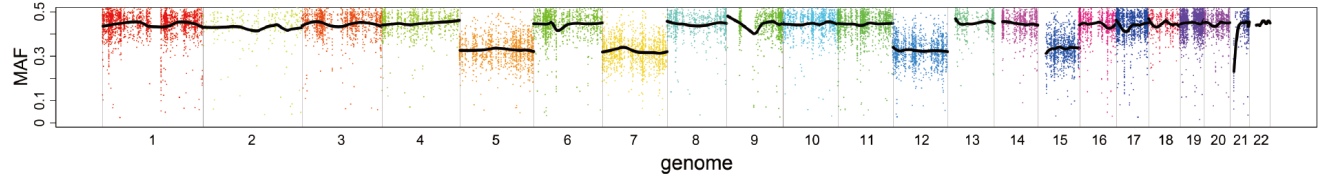

LOVO-SN38

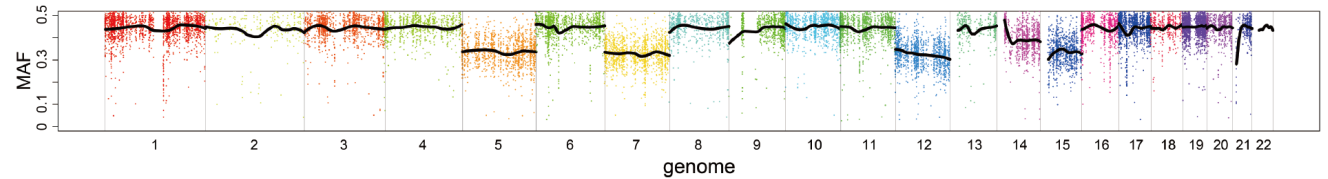


Figure S2

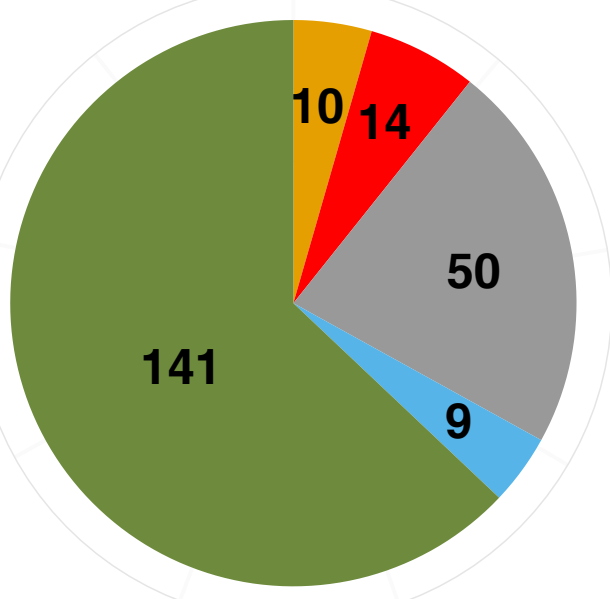

\section{Group}

Cytoplasmic

Extracellular

Mitochondrial

Nuclear

Plasmamembrane 


\section{Supporting information of supplementary tables.}

Table S1. The sequencing depth for the two CRC cell lines with non- and drug-resistance based on NGS data by WES.

\begin{tabular}{|c|c|c|c|c|c|c|}
\hline \multirow[b]{2}{*}{ Chromosome NO. } & \multicolumn{6}{|c|}{ Relative depth value of the six CRC cell lines } \\
\hline & HCT116 & $\begin{array}{c}\text { HCT116- } \\
\text { OxPt }\end{array}$ & $\begin{array}{c}\text { HCT116- } \\
\text { SN38A }\end{array}$ & LoVo & LoVo-OxPt & $\begin{array}{l}\text { LoVo- } \\
\text { SN38A }\end{array}$ \\
\hline 1 & 93.76 & 89.45 & 89.63 & 87.52 & 84.59 & 82.89 \\
\hline 2 & 97.14 & 91.39 & 93.47 & 88.82 & 82.86 & 83.75 \\
\hline 3 & 102.89 & 104.49 & 98.92 & 95 & 90.02 & 88.92 \\
\hline 4 & 100.1 & 92.77 & 95.29 & 90.6 & 82.54 & 85.38 \\
\hline 5 & 100.14 & 94.89 & 95.78 & 119.84 & 127.65 & 126.65 \\
\hline 6 & 103.4 & 97.18 & 97.59 & 95.29 & 90.41 & 90.31 \\
\hline 7 & 87.33 & 83.67 & 84.06 & 118.58 & 115.66 & 113.29 \\
\hline 8 & 115.49 & 111.29 & 110.79 & 82.75 & 79.66 & 79.92 \\
\hline 9 & 88.96 & 85.93 & 86.36 & 82.9 & 80.91 & 78.71 \\
\hline 10 & 104 & 99.27 & 99.66 & 84.73 & 81.01 & 80.78 \\
\hline 11 & 99.56 & 96.16 & 94.63 & 93.4 & 90.21 & 87.64 \\
\hline 12 & 97.38 & 92.25 & 93.93 & 120.02 & 128.33 & 126.77 \\
\hline 13 & 100.96 & 94.25 & 105.72 & 90.25 & 82.4 & 85.81 \\
\hline 14 & 99.07 & 94.4 & 99.34 & 98.62 & 88.69 & 127.6 \\
\hline 15 & 86.94 & 82.24 & 82.45 & 121.36 & 113 & 112.71 \\
\hline 16 & 85.39 & 83.51 & 80.1 & 78.58 & 82.49 & 75.9 \\
\hline 17 & 108.28 & 100.89 & 103.16 & 87.25 & 88.9 & 83.39 \\
\hline 18 & 96.87 & 90.46 & 91.6 & 89.79 & 83.21 & 85.97 \\
\hline 19 & 90.7 & 90.21 & 87.46 & 88.24 & 90.47 & 84.1 \\
\hline 20 & 94.94 & 93.19 & 99.37 & 88.66 & 90.08 & 85.02 \\
\hline 21 & 90.49 & 86.72 & 88.14 & 94.79 & 82.87 & 80.57 \\
\hline 22 & 80.55 & 78.57 & 77.52 & 77.39 & 81.14 & 74.56 \\
\hline$X$ & 51.34 & 48.67 & 48.53 & 47.18 & 43.68 & 44.13 \\
\hline $\mathrm{Y}$ & 0.36 & 0.62 & 0.41 & 12.88 & 4.56 & 11.81 \\
\hline
\end{tabular}


Table S2. Average genome copy numbers of HCT116 and LoVo derived from database

\begin{tabular}{|c|c|c|}
\hline Chromosome NO. & $\begin{array}{c}\text { Average Copy number of } \\
\text { HCT116 }\end{array}$ & $\begin{array}{c}\text { Average Copy number of } \\
\text { LoVo }\end{array}$ \\
\hline 1 & 2 & 2 \\
\hline 2 & 2 & 2 \\
\hline 3 & 2 & 2 \\
\hline 4 & 1.99 & 2 \\
\hline 5 & 2 & 3 \\
\hline 6 & 2 & 2 \\
\hline 7 & 2 & 3 \\
\hline 8 & 2.54 & 2 \\
\hline 9 & 2 & 2 \\
\hline 10 & 2.2 & 2 \\
\hline 11 & 2 & 2 \\
\hline 12 & 2 & 3 \\
\hline 13 & 2 & 2 \\
\hline 14 & 2.01 & 2.01 \\
\hline 15 & 2 & 3 \\
\hline 16 & 2.09 & 2 \\
\hline 17 & 2.46 & 2 \\
\hline 18 & 2 & 2 \\
\hline 19 & 2 & 2 \\
\hline 20 & 2 & 2 \\
\hline 21 & 2 & 2 \\
\hline 22 & 2 & 2.02 \\
\hline $\mathrm{X}$ & 1 & 1.02 \\
\hline $\mathrm{Y}$ & 0 & 1.43 \\
\hline Summary & 46.29 & 50.48 \\
\hline
\end{tabular}


Table S5. The correlation of CRC cell lines and iTRAQ labelling tags.

\begin{tabular}{ccc}
\hline iTRAQ tags & Groups & Replication in a cell line (HCT116 or LoVo) \\
\hline 113 & HCT116 or LoVo & Replication 1 for non-resistant cell line \\
114 & HCT116-OxPt or LoVo-OxPt & Replication 1 for OxPt-drug resistant cell line \\
115 & HCT116-SN38 or LoVo-SN38 & Replication 1 for SN38-drug resistant cell line \\
116 & HCT116 or LoVo & Replication 2 for non-resistant cell line \\
117 & HCT116-OxPt or LoVo-OxPt & Replication 2 for OxPt-drug resistant cell line \\
118 & HCT116-SN38 or LoVo-SN38 & Replication 2 for SN38-drug resistant cell line \\
\hline
\end{tabular}

